\title{
A novel image registration method for InISAR 3D imaging
}

\author{
Zibo Zhou ${ }^{1, a}$, Libing Jiang ${ }^{1}$ and Zhuang Wang ${ }^{1}$ \\ ${ }^{1}$ College of Electronic Science, National University of Defense Technology, 410073 Changsha, China.
}

\begin{abstract}
Image registration is a key intermediate step for Interferometric Inverse Synthetic Aperture Radar (InISAR) three-dimensional (3D) imaging. It arranges the same scatterers of the target on the same pixel cell in different ISAR images, which makes the interferometric processing carried on between the same scatterers to obtain its 3D coordinates. This paper proposes a novel ISAR image registration method of three steps. Firstly, chirp Fourier transform is used to estimate the rotational angular velocity of the target. Secondly, the compensation phase is constructed, according to the rotational angular velocity, to eliminate the wave path difference between different radars echoes. Finally, two-dimensional (2D) Fourier transform is used to yield registered ISAR images. The proposed method achieves the ISAR image registration through phase compensation in echo field, therefore, no extra computation is needed in image field. The experiment results demonstrate the advantages of the proposed method in precision, computation efficiency and practicability.
\end{abstract}

\section{Introduction}

Radar imaging is a hot subject in radar signal processing. With the higher requirements for target recognition and the development of modern wideband high-resolution radar, Interferometric Inverse Synthetic Aperture Radar (InISAR) 3D imaging has gradually become a hot issue in the field of radar imaging [1-4].

During the InISAR 3D imaging processing, ISAR image registration is a key intermediate step, whose purpose is to arrange the same scatterers of the target on the same pixel cell in different images and ensure that the interferometric process is performed between the same scatterers. Since the interferometric phase difference contains the coordinate information of the scatterers, the 3D structure of the target can be obtained [5].

There are many literatures and applications concerning ISAR image registration and InISAR 3D imaging techniques. The correlation coefficient based image registration method, which was widely used for SAR image registration, was firstly introduced to solve the ISAR image registration problem [6,7]. Wang et al. [8] presented a SAR image registration method based on integrating real and complex correlation function. Through coregistration sensitive factor, the appropriate correlation function is selected to measure the quality of image registration. However, the targets observed by SAR and ISAR are different. And the target of ISAR only account for a small part of the ISAR image, so the correlation coefficient is easily affected by noise. Zhang et al. [9] proposed a multi-antenna configuration InISAR imaging system, which arranges multiple antennas along two vertical baseline directions. The antennas on the larger baseline are used to estimate the rotational angle, while the antennas on the smaller baseline are used for ISAR imaging. By using the wave path difference calculated by the rotational angle to compensate the echoes, the mismatch between the ISAR images is eliminated. However, the five-antenna imaging system increases the cost of hardware.

Aiming at the high precision and low hardware cost, this paper proposes a novel image registration method based on wave path difference compensation. Firstly, the chirp Fourier transform is used to estimate the rotational angular velocity of the target relative to the radar. Then the wave path compensation phase is constructed and the echo is compensated to eliminate the mismatch between ISAR images.

The rest of this paper is organized as follows: Section II analyses the L-shaped three-antenna InISAR 3D image model. Section III is devoted to the ISAR image registration method based on wave path difference compensation. Section IV introduces the simulation experiment result. Finally, Section V draws conclusion on the proposed imaging method.

\section{L-shaped InISAR 3D image model}

The geometric configuration of the L-shaped threeantenna InISAR imaging system is shown in Figure 1, which contains two orthogonal coordinate systems in parallel, i.e., the radar coordinate system (A-XYZ) and the target coordinate system $(\mathrm{O}-\mathrm{xyz})$. The locations of three radars in radar coordinate system are $A(0,0,0)$, $B(0,0, L)$ and $C(L, 0,0)$ respectively. Only radar A

\footnotetext{
a Corresponding author: zibo_travel@163.com
} 
transmits electromagnetic wave and all the three radars receive echo pulses from the target. The baselines $\mathrm{AB}$ and $\mathrm{AC}$ are perpendicular to each other and they have the same length, denoted as $L$. The origin (i.e., reference point) of the target coordinate system is located at the geometric centre of the target at position $O\left(X_{0}, Y_{0}, \mathrm{Z}_{0}\right)$ in the radar coordinate system. $\hat{t}$ is the imaging fast-time, and $t_{m}$ is the imaging slow-time. The distance from $O$ to radar $\mathrm{A}, \mathrm{B}$, and $\mathrm{C}$ is $R_{O A}, R_{O B}$ and $R_{O C}$, respectively, which are functions of slow-time $t_{m} . P$ is the $k$ th scatterer on the target, which position in target coordinate system is $\left(x_{k}, y_{k}, z_{k}\right)$. The distance from $P$ to $\operatorname{radar} \mathrm{A}, \mathrm{B}$, and $\mathrm{C}$ is $R_{A k}, R_{B k}$ and $R_{C k}$ respectively. And the motion velocity of the target along $\overline{A O}, \overline{B O}$ and $\overline{C O}$ directions is $V_{A k}, V_{B k}$ and $V_{C k}$, respectively.

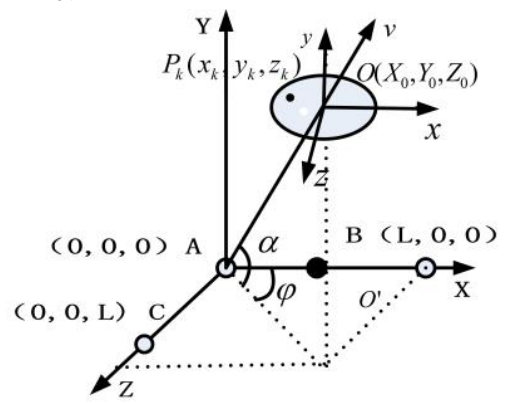

Figure 1. Configuration of L-shape three-antenna InISAR imaging system

Denoting that the radar transmits Linear Frequency Modulation (LFM) signal with the carrier frequency $f_{c}$, bandwidth $B$, pulse duration time $T_{p}$, pulse interval $T_{r}$, dwell time $T_{1}$, and the scattering intensity $\sigma_{k}$ for the $k$ th scatterer. And $c$ means the light velocity, the wavelength is $\lambda$, the chirp rate is $\gamma$. All the three radars generally use the same reference distance $R_{\text {refa }}$ to dechirp. Denoting $R_{\Delta A k}=R_{A k}-R_{r e f A}, R_{\Delta B k}=R_{B k}-R_{\text {refA }}, R_{\Delta C k}=R_{C k}-R_{\text {refA }}$ and $R_{\text {refA }}=R_{A O}$.

InISAR imaging technology is a combination of highresolution ISAR imaging and interferometric technique. Therefore, high-resolution ISAR image is the precondition of the InISAR 3D imaging. According to the existing motion compensation and pulse compression methods, high-resolution ISAR images can be obtained. The high-resolution ISAR imaging results obtained by the three radars can be written as:

$S_{A}\left(f, f_{m}\right)=\sum \sigma_{k} T_{p} T_{1} \sin c\left[T_{p}\left(f_{i}-\frac{2 \gamma}{c} R_{\Delta A k}\right)\right] \sin c\left[T_{1}\left(f_{m}-\frac{2 V_{A k}}{\lambda}\right)\right] \exp \left(-j \frac{4 \pi R_{\Delta A k 0}}{\lambda}\right)$

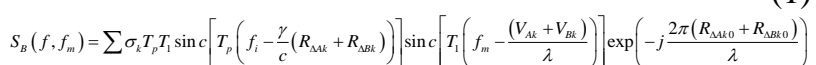

$S_{c}\left(f, f_{m}\right)=\sum \sigma_{k} T_{p} T_{1} \sin c\left[T_{p}\left(f_{i}-\frac{\gamma}{c}\left(R_{\Delta \mu k}+R_{\Delta c k}\right)\right)\right] \operatorname{sinc}\left[T_{1}\left(f_{m}-\frac{\left(V_{\Delta k}+V_{c k}\right)}{\lambda}\right)\right] \exp \left(-j \frac{2 \pi\left(R_{\Delta \mu k}+R_{\Delta c c_{0}}\right)}{\lambda}\right)$

Where $f$ is the frequency relative to fast-time, $f_{m}$ is the Doppler frequency relative to slow-time.
From (1) and (2), the mismatch for the $k$ th scatterer between ISAR images $S_{A}\left(f, f_{m}\right)$ and $S_{B}\left(f, f_{m}\right)$ is:

$$
\begin{gathered}
\Delta f=\frac{\gamma}{c}\left(R_{\Delta A k 0}-R_{\Delta B k 0}\right)=\left.\frac{\gamma}{c}\left(R_{\Delta A k}-R_{\Delta B k}\right)\right|_{t_{m}=0}=\left.\frac{\gamma}{c}\left(R_{A k}-R_{B k}\right)\right|_{t_{m}=0} \\
\Delta f_{m}=\frac{V_{A k}-V_{B k}}{\lambda}=\frac{\left.\left(R_{A k}-R_{B k}\right)\right|_{t_{m}=T_{1}}-\left.\left(R_{A k}-R_{B k}\right)\right|_{t_{m}=0}}{\lambda T_{1}}
\end{gathered}
$$

After ISAR image registration, interferometric technique is introduced to obtain the $3 \mathrm{D}$ structure of the target. The relationship between the interferometric phase difference and the coordinates are:

$$
\begin{aligned}
& \Delta \varphi_{A B}=-\varphi_{A}+\varphi_{B}=\frac{2 \pi}{\lambda}\left(R_{\Delta A k 0}-R_{\Delta B k 0}\right)=\frac{2 \pi}{\lambda} \frac{2 L\left(X_{0}+x_{k}\right)-L^{2}}{R_{A k}+R_{B k}} \\
& \Delta \varphi_{A C}=-\varphi_{A}+\varphi_{C}=\frac{2 \pi}{\lambda}\left(R_{\Delta A k 0}-R_{\Delta C k 0}\right)=\frac{2 \pi}{\lambda} \frac{2 L\left(Y_{0}+z_{k}\right)-L^{2}}{R_{A k}+R_{B k}}
\end{aligned}
$$

\section{The proposed ISAR image registration method}

\subsection{Estimation of wave path difference}

From (4) and (5), it can be seen that if the radars B and C use the same reference distance as $R_{A O}$, which is the exact distance from the target to radar $\mathrm{A}$, the mismatch between ISAR images is serious. However, if the reference distances of radar B and radar $\mathrm{C}$ were manipulated to $R_{B O}$ and $R_{C O}$ respectively, the mismatch would be alleviated effectively. Namely, $R_{\triangle B k}^{\prime}=R_{B k}-R_{B O}$, $R_{\triangle C k}^{\prime}=R_{C k}-R_{C O}$. Then, the mismatch in range and crossrange can be written as follow:

$$
\begin{aligned}
\Delta f^{\prime} & =\frac{\gamma}{c}\left(R_{\Delta A k 0}-R_{\Delta B k 0}^{\prime}\right)=\left.\frac{\gamma}{c}\left[\left(R_{A k}-R_{B k}\right)-\left(R_{A O}-R_{B O}\right)\right]\right|_{t_{=0}} \\
& <\left.\frac{\gamma}{c}\left[\left(R_{A k}-R_{B k}\right)-\left(R_{A O}-R_{A O}\right)\right]\right|_{t_{m}=0}=\left.\frac{\gamma}{c}\left[R_{A k}-R_{B k}\right]\right|_{t_{m}=0} \\
\Delta f_{m}^{\prime} & =\frac{1}{\lambda}\left[\left.\left(R_{\Delta A k}-R_{\Delta B k}^{\prime}\right)\right|_{t_{m}=T_{1}}-\left.\left(R_{\Delta A k}-R_{\Delta B k}^{\prime}\right)\right|_{t_{m}=0}\right] \\
& =\frac{1}{\lambda}\left\{\left.\left[\left(R_{A k}-R_{B k}\right)-\left(R_{A O}-R_{B O}\right)\right]\right|_{t_{m}=T_{1}}-\left.\left[\left(R_{A k}-R_{B k}\right)-\left(R_{A O}-R_{B O}\right)\right]\right|_{t_{m}=0}\right\} \\
& <\frac{1}{\lambda}\left\{\left.\left[\left(R_{A k}-R_{B k}\right)-\left(R_{A O}-R_{A O}\right)\right]\right|_{t_{m}=T_{1}}-\left.\left[\left(R_{A k}-R_{B k}\right)-\left(R_{A O}-R_{A O}\right)\right]\right|_{t_{m}=0}\right\} \\
& =\frac{1}{\lambda}\left[\left.\left(R_{\Delta A k}-R_{\Delta B k}\right)\right|_{t_{m}=T_{1}}-\left.\left(R_{\Delta A k}-R_{\Delta B k}\right)\right|_{t_{m}=0}\right]
\end{aligned}
$$

Consequently, the wave path difference that radar B and $\mathrm{C}$ needs compensating is:

$$
\begin{aligned}
& \Delta R_{m}^{B}=R_{A O}-R_{B O} \\
& \Delta R_{m}^{C}=R_{A O}-R_{C O}
\end{aligned}
$$

Furthermore, the compensation phase corresponding to the abovementioned wave path difference in (10) and (11) should be constructed to compensate the received echoes of radar $\mathrm{B}$ and $\mathrm{C}$, which are as follow:

$$
\begin{aligned}
& P h a_{m}^{B}=\exp \left\{-j 2 \pi \gamma \hat{t} \Delta R_{m}^{B} / c\right\} \exp \left\{-j 2 \pi \Delta R_{m}^{B} / \lambda\right\} \\
& P h a_{m}^{C}=\exp \left\{-j 2 \pi \gamma \hat{t} \Delta R_{m}^{C} / c\right\} \exp \left\{-j 2 \pi \Delta R_{m}^{C} / \lambda\right\}
\end{aligned}
$$

Based on the above analysis, the main reason for the mismatch of the ISAR images is the wave path difference from the scatterer to different radar. Therefore, the main purpose of this section is to estimate and compensate the 
wave path difference. And a wave path difference estimation method based on angle motion parameter estimation is proposed.

According to the derivation in Section III in [9], the difference between $R_{A O}\left(t_{m}\right)$ and $R_{B O}\left(t_{m}\right)$ can be modelled as:

$$
\Delta R_{m}^{B}=R_{A O}\left(t_{m}\right)-R_{B O}\left(t_{m}\right)=L \sin \left(\theta\left(t_{m}\right)-\theta\left(t_{0}\right)\right) \approx L \bullet w^{A B} t_{m}
$$

Similarly, the reference distance difference between radar $\mathrm{A}$ and radar $\mathrm{C}$ is $\Delta R_{m}^{C} \approx L \bullet w^{A C} t_{m}$.

Where the rotational angular velocities $w^{A B}$ and $w^{A C}$ are the projections of real rotational angular velocity $w$ in XOY plane and ZOY plane.

Consequently, the ISAR image registration problem is transformed from the problem of wave path difference estimation to the problem of target rotational angle estimation.

\subsection{Estimation of rotational angle based on chirp Fourier transform.}

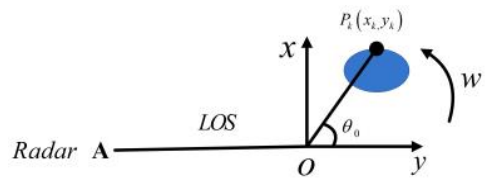

Figure 2. Turntable model of ISAR imaging

Based on the turntable model in Figure 2, for the targets with uniform rotational angular velocity relative to radar A, the distance from scatterer $P_{k}$ to radar A is:

$$
\begin{aligned}
R_{A k} & =R_{A O}+R_{\triangle A k}=R_{A O}+R_{O k} \cos \left(\theta_{0}+w t_{m}\right) \\
& =R_{A O}+\sqrt{x_{k}^{2}+y_{k}^{2}}\left(\cos \theta_{0} \cos w t_{m}-\sin \theta_{0} \sin w t_{m}\right) \\
& =R_{A O}+y_{k} \cos w t_{m}-x_{k} \sin w t_{m}
\end{aligned}
$$

Then, after eliminating remaining video phase and envelope skew, the range profile for radar $\mathrm{A}$ is:

$$
S_{A}\left(f, t_{m}\right)=\sum \sigma_{k} T_{p} \sin c\left(T_{p}\left(f+\frac{2 \gamma}{c} R_{\Delta A k}\right)\right) \exp \left(-j \frac{4 \pi}{\lambda} R_{\Delta A k}\right) \operatorname{rect}\left(\frac{t_{m}}{T_{1}}\right)(16)
$$

Where $R_{\Delta A k}=y_{k} \cos w t_{m}-x_{k} \sin w t_{m}$, then the Doppler phase in (16) is:

$$
\text { Phase }_{d i}=\exp \left\{-j \frac{4 \pi\left(y_{k} \cos w t_{m}-x_{k} \sin w t_{m}\right)}{\lambda}\right\}
$$

Consequently, when the rotational angle is small, the Doppler frequency can be obtained as follow:

$$
f_{d c i}=\frac{1}{2 \pi} \frac{d \text { Phase }_{d i}}{d t_{m}}=-\frac{2 w}{\lambda}\left(y_{k} \sin w t_{m}+x_{k}\right) \approx-\frac{2 w}{\lambda} x_{k}-\frac{2 y_{k} w^{2}}{\lambda} t_{m}
$$

From (18), the signal in the slow-time domain is LFM signal, and the chirp rate is:

$$
k=-\frac{2 y_{k} w^{2}}{\lambda}
$$

Equation (19) indicates that rotational angular velocity $w$ is the dependent variable of the chirp rate $k$, given the wave length $\lambda$ and the scatterer coordinate $y_{k}$. In other words, it should be noted that the estimation of the rotational angular velocity is equivalent to the estimation of the chirp rate in the slow-time domain. Hence the chirp Fourier transform is introduced as an efficient tool to estimate the chirp rate $k$. The chirp Fourier transform of range profile is defined as:

$$
F(f, \gamma)=\int S_{A}\left(f, t_{m}\right) \exp \left[j 2 \pi \bullet \frac{1}{2} \gamma t_{m}{ }^{2}\right] d t_{m}
$$

Where the scope of the compensated chirp rate $\gamma$ is:

$$
\gamma=\frac{2 y_{k} w}{\lambda} \in\left\{\frac{2 y_{k} w_{\min }}{\lambda}, \frac{2 y_{k} w_{\max }}{\lambda}\right\}
$$

For each chirp rate $\gamma$ in the scope, the entropy corresponding to its chirp Fourier transform result $F(f, \gamma)$ is calculated. Then, the chirp rate parameter $\gamma$, whose chirp Fourier transform result has the minimum entropy, is selected as the parameter estimation result of chirp rate of the echo Doppler frequency. The definition of entropy is as written in Section IV in [10]. Furthermore, to obtain a more robust chirp rate parameter estimation result, the range profiles of the range cells around the strong scattering range cell are selected as the range profile block (the change of the chirp rate is little), which is used to estimate the chirp rate. After obtaining the chirp rate of each range profile block, according to the linear relationship between the chirp rate and the distance $y_{k}$ of the scatterer, as is shown in (19), the least squares fit method is used to fit the chirp rates obtained from the multiple range profile blocks. And the relationship between slope $k$ of the fitted line and the rotational angular velocity is:

$$
w=\sqrt{|k| \lambda / 2}
$$

From the above, the flowchart of the whole target rotational angular velocity estimation algorithm is shown in Figure 3.

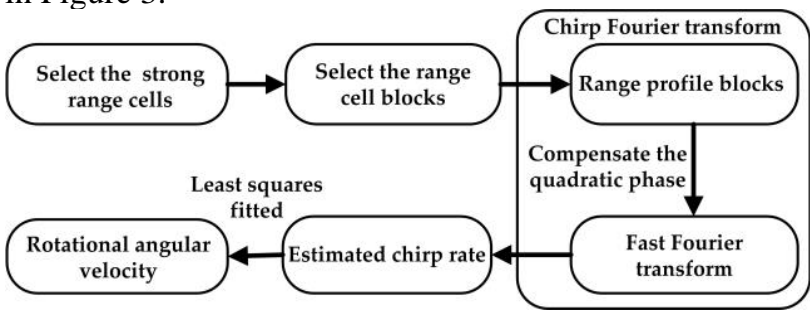

Figure 3. Flowchart of the rotational angular velocity estimation algorithm

\subsection{Image registration based on wave path difference compensation.}

After the rotational angular velocity estimation, the compensation phase is constructed to eliminate the effect of wave path difference on image mismatch. Hence, the concrete procedure of InISAR 3D imaging is as follows:

1) Estimate the rotational angular velocity based on chirp Fourier transform;

2) Calculate the wave path difference between centroid of target and different radars: $L \bullet w^{A B} t_{m}$ and $L \bullet w^{A C} t_{m}$;

3) Implement wave path difference compensation for the received echoes of radar $\mathrm{B}$ and radar $\mathrm{C}$;

4) Yield the ISAR images of target without mismatch;

5) Implement interferometric between registered ISAR images to form the 3D structure of the target. 


\section{Experiment}

In this section, the ISAR image registration method based on wave path difference compensation is validated and compared with the method based on correlation coefficient. The configuration of InISAR imaging is shown in Figure 1 , the baseline $L$ is $10 \mathrm{~m}$. The parameters of the radar system and the target are shown in Table 1.

Table.1 The parameters of radar and target

\begin{tabular}{ccc}
\hline \multirow{3}{*}{ Radar } & Carrier frequency $f_{c}$ & $10 \mathrm{GHz}$ \\
\cline { 2 - 3 } & Pulse duration $T_{P}$ & $0.1 \mathrm{~ms}$ \\
\cline { 2 - 3 } & Bandwidth B & $1 \mathrm{GHz}$ \\
\cline { 2 - 3 } & Pulse Repeat Frequency & $100 \mathrm{~Hz}$ \\
\cline { 2 - 3 } Target & Pulse number & 500 \\
\cline { 2 - 3 } & Scatterer number & 8 \\
\cline { 2 - 3 } & Rotational angular velocity & $0.0112 \mathrm{rad} / \mathrm{s}$ \\
\hline
\end{tabular}

\subsection{Result of the image registration based on wave path difference compensation.}

The estimation results of the echo Doppler chirp rate $\gamma$ are shown in Figure 4, in which the blue dotted line shows the estimated results of the chirp Fourier transform for each range profile block, and the red line is the least squares fitted result. Then, the estimation result of the rotational angular velocity $w$ is: $w=\sqrt{-\lambda k / 2}=0.0111$.

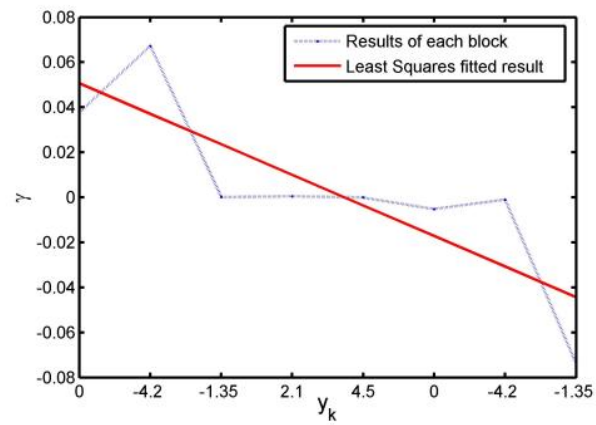

Figure 4. Estimation results of the Doppler chirp rate

As is shown in Figure 5, the range profiles of three radars are of high similarity, which indicates that the mismatch along range direction is eliminated. After pulse compression along cross-range direction, the ISAR images of the target are obtained. For convenience sake, the positions of the strong scatterers in the ISAR images can be extracted in Table 2. The first number in parentheses is scatterer position along cross-range cell, and the second one is the position along range cell. As can be seen from the Table 2, the positions of the strong scatterers in the three ISAR images of three different radars are identical, namely, the mismatch between the ISAR images is eliminated. In other words, image registration is accomplished.

Since the ISAR image registration is the intermedium step of the interferometric ISAR imaging process, hence the ultimate InISAR 3D imaging results should be used to measure the performance of the proposed method. According to Section 3.3, the $x$ and $z$ coordinates of the scatterer can be obtained. And the $y$ coordinate is relative to the distance of scatterer along range direction. The 3D imaging result is shown in Figure 6, the blue dots indicate the true structure of the target, and the red circles indicate the 3D imaging result. The average coordinates $(x, y, z)$ difference between the red circle and the relative blue dot is 0.3034 . It is visible that the proposed method can effectively implement ISAR image registration of an L-shaped three-antenna InISAR imaging system. More importantly, InISAR 3D imaging works well.

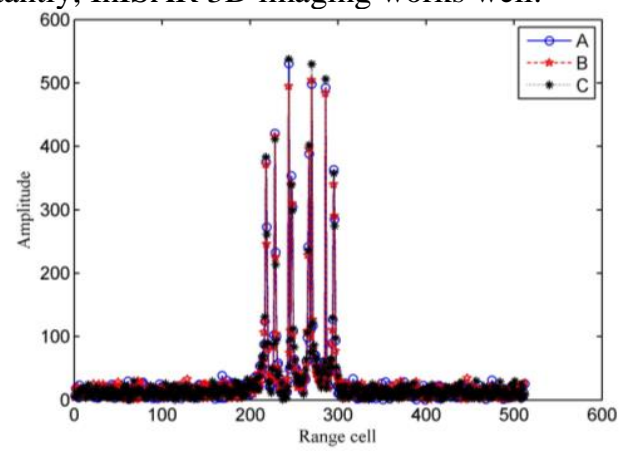

Figure 5. Range profiles without mismatch

Table 2. The location of scatterer in ISAR images

\begin{tabular}{cccc}
\hline Number & A & B & C \\
\hline 1 & $(247,218)$ & $(247,218)$ & $(247,218)$ \\
\hline 2 & $(279,229)$ & $(279,229)$ & $(279,229)$ \\
\hline 3 & $(229,243)$ & $(229,243)$ & $(229,243)$ \\
\hline 4 & $(260,248)$ & $(260,248)$ & $(260,248)$ \\
\hline 5 & $(253,267)$ & $(253,267)$ & $(253,267)$ \\
\hline 6 & $(242,269)$ & $(242,269)$ & $(242,269)$ \\
\hline 7 & $(256,286)$ & $(256,286)$ & $(256,286)$ \\
\hline 8 & $(244,295)$ & $(244,295)$ & $(244,295)$ \\
\hline
\end{tabular}
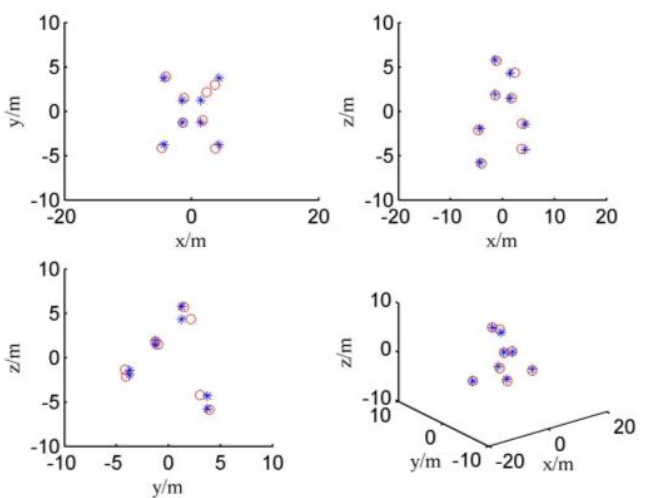

Figure 6. InISAR imaging result through the proposed method

\subsection{The experiment results of the image registration based on correlation coefficient.}

As is shown in Figure 7, the correlation coefficient between image $\mathrm{A}$ and image $\mathrm{B}$ and image $\mathrm{C}$ are calculated. The eclipse time of the correlation between image A and image B is $15916 \mathrm{~s}$, and that between image $\mathrm{A}$ and image $\mathrm{C}$ is 15740 s. The simulation was conducted on MATLAB software on computer configured with an Intel(R) Core ${ }^{\mathrm{TM}}$ i7 $7700 \mathrm{CPU} @ 3.6 \mathrm{GHz}$ and $16 \mathrm{~GB}$ RAM. From the results in Figure 7 , the optimal shift 
amount of the image $\mathrm{B}$ is 0.8 pixels along range direction and 18.3 pixels along cross-range direction, and that of the image $\mathrm{C}$ is 0.7 pixels along range direction and 10.8 pixels along cross-range direction. Image $\mathrm{B}$ and image $\mathrm{C}$ is shifted, respectively, based on the optimal shift amount of $\mathrm{B}$ and $\mathrm{C}$. Additionally, the positions of strong scatterers are extracted in Table 3. It is obvious that there is still mismatch between ISAR images of different radars. Therefore, the registration accuracy of the registration method based on the correlation coefficient is poor. And, finally, InISAR 3D imaging is inefficient with correlation-based method, as shown in Figure 8. The average coordinates $(x, y, z)$ difference between the red circle and the relative blue dot is 45.8529 .

In conclusion, the method based on wave path difference compensation can effectively complete the ISAR image registration, and the InISAR imaging quality of it is better than that of the correlation-based method.

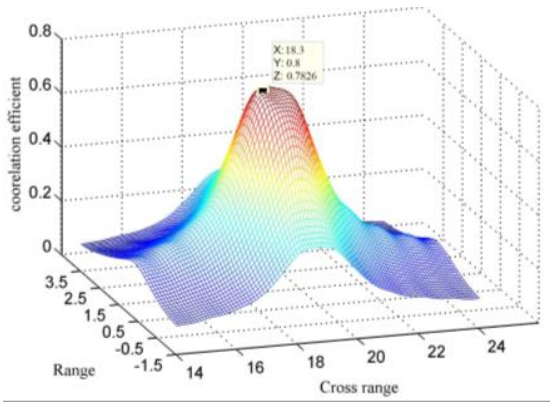

a

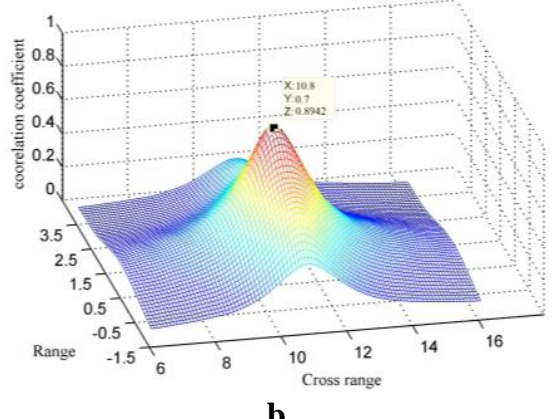

Figure 7. Distribution of correlation coefficients during registration;

(a) Radar B (range 0.8, cross-range 18.3, eclipse time:15916s),

(b) Radar C (range 0.7, cross-range 10.8, eclipse time:15740s).

Table 3. The mismatched scatterer in ISAR image

\begin{tabular}{cccc}
\hline Number & A & B & C \\
\hline 1 & $(247,218)$ & $(246,218)$ & $(247,218)$ \\
\hline 2 & $(279,229)$ & $(278,229)$ & $(278,230)$ \\
\hline 3 & $(229,243)$ & $(229,243)$ & $(229,243)$ \\
\hline 4 & $(260,248)$ & $(260,247)$ & $(260,248)$ \\
\hline 5 & $(253,267)$ & $(252,266)$ & $(253,267)$ \\
\hline 6 & $(242,269)$ & $(242,269)$ & $(241,270)$ \\
\hline 7 & $(256,286)$ & $(256,286)$ & $(256,286)$ \\
\hline 8 & $(244,295)$ & $(243,295)$ & $(244,295)$ \\
\hline
\end{tabular}
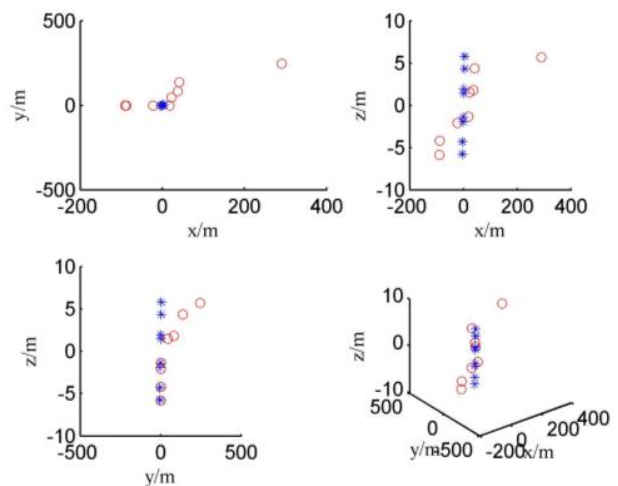

Figure 8. InISAR imaging result through the method based on correlation efficient

\section{Conclusion}

In this paper, a novel ISAR image registration method based on wave path difference compensation is proposed. The proposed method eliminates the wave path difference between the scatterer and different radars by the wave path difference phase compensation in echo field, and achieves ISAR image registration. In comparison to the ISAR image registration method based on correlation coefficient, the proposed method is more precise and more efficient significantly. In brief, the proposed ISAR image registration method based on wave path difference compensation has advantages in precision and computing efficiency, compared with precedent methods.

\section{References}

1. Wang, G., Xia, X., Chen Victor, C.: Threedimensional ISAR imaging of maneuvering targets using three receivers, IEEE Trans. Image Process., 10, (3), pp. 436-447, 2001.

2. Tian B, Zou J, Xu S, et al. Squint model interferometric ISAR imaging based on respective reference range selection and squint iteration improvement[J]. Radar Sonar \& Navigation Iet, 9(9):1366-1375, 2015.

3. Chen C C, Andrews H C. Target-Motion-Induced Radar Imaging $[\mathrm{J}]$. Aerospace \& Electronic Systems IEEE Transactions on, AES-16(1):2-14, 1980.

4. Martorella M, Stagliano D, Salvetti F, et al. 3D interferometric ISAR imaging of noncooperative targets $[\mathrm{J}]$. Aerospace \& Electronic Systems IEEE Transactions on, 50(4):3102-3114, 2014.

5. Tian B, Li N, Liu Y, et al. A novel image registration method for InISAR imaging system[C] Millimetre Wave and Terahertz Sensors and Technology VII. International Society for Optics and Photonics, 2014.

6. Stagliano D, Lischi S, Massini R, et al. Soft 3DISAR image reconstruction using a dual interferometric radar [C] Radar Conference. IEEE:0572-0576, 2015.

7. Nasirian M, Bastani M H. A Novel Model for ThreeDimensional Imaging Using Interferometric ISAR in Any Curved Target Flight Path[J]. IEEE Transactions on Geoscience \& Remote Sensing, 52(6):3236-3245, 2014. 
8. Wang Q, Jishuang Q U, Huang H, et al. A Method Based on Integrating Real and Complex Correlation Function for InSAR Image Coregistration[J]. Acta Geodaetica Et Cartographica Sinica, 2012.

9. Zhang Q, Yeo T S, Du G, et al. Estimation of threedimensional motion parameters in interferometric ISAR imaging $[\mathrm{J}]$. Geoscience \& Remote Sensing IEEE Transactions on, 42(2):292-300, 2004.

10. $\mathrm{Wu} \mathrm{W}, \mathrm{Hu} \mathrm{P}, \mathrm{Xu} \mathrm{S}$, et al. Image registration for InISAR based on joint translational motion compensation $[\mathrm{J}]$. Iet Radar Sonar \& Navigation, 11(10):1597-1603, 2017. 